\title{
BMJ Open Effect modification by gender of the influence of obstructive sleep apnoea characteristics on dyslipidaemia in China: a cross-sectional study
}

\author{
Yunyan Xia, ${ }^{\oplus, 2,3,4}$ Juanjuan Zou, ${ }^{1,3,4}$ Huajun Xu, ${ }^{1,3,4}$ Hongliang Yi, ${ }^{1,3,4}$ Jian Guan, ${ }^{1,3,4}$ \\ Shankai Yin ${ }^{1,3,4}$
}

To cite: Xia Y, Zou J, Xu H, et al. Effect modification by gender of the influence of obstructive sleep apnoea characteristics on dyslipidaemia in China: a crosssectional study. BMJ Open 2019;9:e028509. doi:10.1136/ bmjopen-2018-028509

- Prepublication history and additional material for this paper are available online. To view these files, please visit the journal online (http://dx.doi. org/10.1136/bmjopen-2018028509).

YX and JZ contributed equally.

Received 11 December 2018 Revised 14 August 2019 Accepted 14 August 2019

A Check for updates

(C) Author(s) (or their employer(s)) 2019. Re-use permitted under CC BY-NC. No commercial re-use. See rights and permissions. Published by BMJ.

For numbered affiliations see end of article.

Correspondence to Dr Huajun Xu; sunnydayxu2010@163.com

Dr Jian Guan; guanjian0606@sina.com

\section{ABSTRACT}

Objectives Obstructive sleep apnoea (OSA)

characteristics differ by gender, possibly affecting any association between OSA and dyslipidaemia. We explored whether gender influenced any association between OSA characteristics and dyslipidaemia.

Methods/Design This was a cross-sectional, largescale hospital-based study. Male and female risks of dyslipidaemia by OSA characteristics were assessed with logistic regression. Additive interactions were measured using three indices: the relative excess risk due to interaction, the attributable proportion due to interaction and the synergy index. Multiplicative interaction was evaluated via logistic regression.

Setting A single secondary-care setting in China.

Participants 3760 patients with OSA.

Primary and secondary outcome measures Outcomes were male and female risks of dyslipidaemia, and the associated additive and multiplicative interactions between the apnoea-hypopnea index (AHI), the oxygendesaturation index (ODI), the microarousal index (MAI), and gender.

Results After controlling for confounding factors, males (but not females) with AHls $>30$ were at higher risk of hyper-total cholesterol (TC), hypo-high-density lipoprotein cholesterol (HDL-C) status and a hyper-TC/HDL-C ratio than males with $\mathrm{AHI} s \leq 30$. Positive additive interactions were evident between the male gender and $\mathrm{AHI}$ on a hyper-TC/HDL-C ratio and hypo-HDL-C status. Males with ODls $>40.1$ were at higher risk of hypo-HDL-C status and a hyper-TC/HDL-C ratio than males with $0 \mathrm{DIs} \leq 40.1$. Positive additive and multiplicative interactions were evident between male gender and ODI on hyper-TC/ $\mathrm{HDL}-\mathrm{C}$ ratio. Males with MAls $>28.6$ were at higher risk of hyper-TC and hyper-low-density lipoprotein cholesterol status than males with MAls $\leq 28.6$, but no statistically significant interactions were apparent between gender and MAl.

Conclusions Males (but not females) with higher AHIs, ODls or MAls were at higher risks of some measures of dyslipidaemia. Positive interactions between male and severe OSA or intermittent hypoxia on some measures of dyslipidaemia were apparent. Thus, dyslipidaemia should be evaluated in patients with OSA, especially males with severe OSA or intermittent hypoxia.
Strengths and limitations of this study

- This cross-sectional study had a large sample size.

- This study is the first to explore (in great detail) the effect modification by gender of obstructive sleep apnoea (OSA) characteristics on dyslipidaemia. We sought both additive and multiplicative interactions between gender and sleep apnoea characteristics.

- Sleep data were collected via standard polysomnography, ensuring reliability of the results.

- Certain potential confounding factors, the observational hospital-based design and the 'first night effect' constitute the major limitations of the study.

\section{INTRODUCTION}

Obstructive sleep apnoea (OSA) is one of the most common sleep disorders; the prevalence of moderate-to-severe OSA in a recent population-based study in Switzerland was $23.4 \%$ in females and $49.7 \%$ in males. ${ }^{1}$ Many studies, including the Sleep Heart Health Study and the PROOF (PROgnostic indicator OF cardiovascular and cerebrovascular events) Study, have found that OSA is independently and positively associated with total cholesterol (TC), triglyceride (TG) and low-density lipoprotein cholesterol (LDL-C) levels, and is negatively associated with high-density lipoprotein cholesterol (HDL-C) levels. ${ }^{2-10}$ The two typical pathophysiological characteristics of OSA, that is, intermittent hypoxia and sleep fragmentation, were also independently associated with dyslipidaemia. ${ }^{89}$

Previous studies have found that OSA prevalence, onset time, severity, symptoms and characteristics differ significantly between males and females. ${ }^{11-14}$ Males are twice as likely as females to develop OSA. Males develop more severe and earlier-onset OSA, and exhibit more typical OSA symptoms (ie, more obvious oxygen desaturation and longer apnoea duration); females are more likely to 
exhibit rapid-eye-movement-dominant events. ${ }^{11-13}$ The cumulative exposure to OSA differs between females and males. ${ }^{1516}$ Also, males and females obviously differ in terms of the physiological cycle; oestrogen maintains female lipid metabolism prior to menopause. Male and female patients with OSA may also differ in terms of lifestyle factors, including alcohol consumption and smoking. Such gender differences may affect the relationships between OSA and metabolic disorders; one study found that OSA was independently associated with liver fat accumulation in males but not females. ${ }^{17}$ Thus, we hypothesised that the relationships between OSA characteristics and dyslipidaemia might differ between males and females. Gender might modify the effects of OSA characteristics on dyslipidaemia.

To date, no study has explored the effect modification by gender of intermittent hypoxia and sleep fragmentation in terms of dyslipidaemia. We thus performed a large-scale cross-sectional study to investigate these issues.

\section{METHODS}

\section{Patient and public involvement}

When formulating the research questions, and when designing the study, the development and content validity of the outcome measure was ensured by qualitatively interviewing patients who underwent polysomnography (PSG). Patients were not involved in the recruitment or conduct of the study. When the results are published, they will be disseminated in a local newspaper and on WeChat.

\section{Study design}

This was a cross-sectional large-scale hospital-based study.

\section{Setting}

From 2010 to 2016, we consecutively enrolled 4583 subjects with suspected OSA treated in the sleep centre of the Department of Otolaryngology/Head and Neck Surgery, Shanghai Jiao Tong University Affiliated Sixth People's Hospital.

\section{Participants}

In total, 823 patients who did not take lipid-lowering drugs were excluded for the following reasons: age less than 18 years $(n=41)$; presence of serious systematic diseases $(e g$, heart or renal failure; $n=4)$; missing data $(n=91)$; abnormal data $(\mathrm{n}=227)$; prior treatment (eg, weight loss surgery, Z-palatopharyngoplasty or continuous positive airway pressure treatment; $\mathrm{n}=158)$; another sleep disorder $(\mathrm{n}=1)$; and failure to meet the diagnostic criteria for OSA $(n=301)$. In total, 3760 subjects were finally included (figure 1). Written informed consent was obtained from all participants according to the guidelines of the National Ethics Regulation Committee.

\section{Anthropometric measurements}

Weight was measured, with the subject standing in light clothing while barefoot. Height was the maximum vertical distance from the bottom of the feet to the highest point on the head while standing barefoot, with the feet together

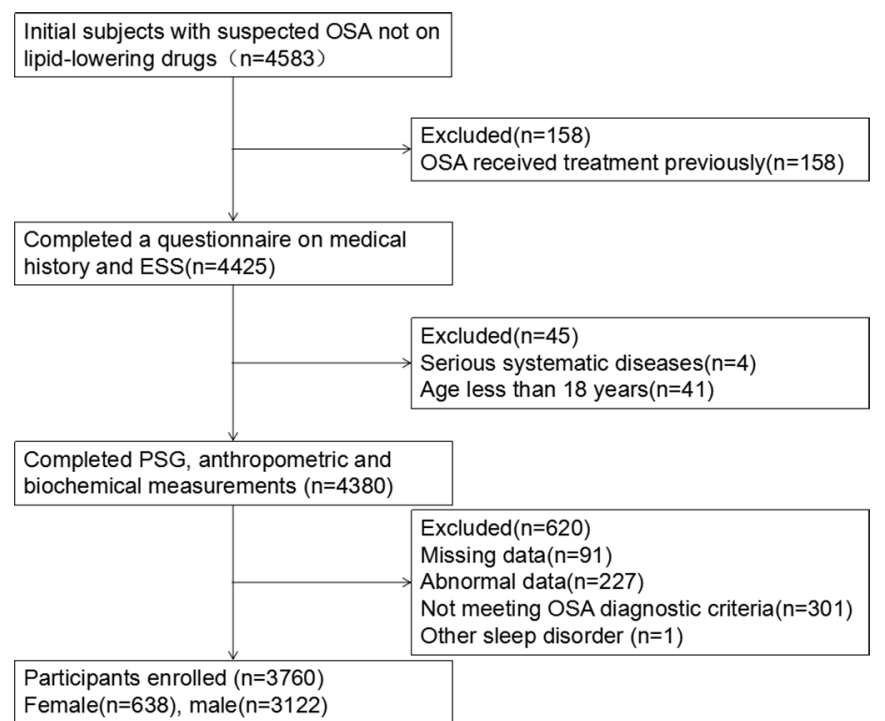

Figure 1 Enrollment flow chart for the study population. ESS, Epworth Sleepiness Scale; OSA, obstructive sleep apnoea; PSG, polysomnography.

and arms by the sides, and with the heels, buttocks and upper back in contact with a wall. Body mass index (BMI) was calculated as weight $(\mathrm{kg}) /$ height $^{2}\left(\mathrm{~m}^{2}\right)$. Waist circumference (WC) was measured midway between the lower costal margin and the iliac crest, and hip circumference (HC) was the maximum girth at the greater trochanters. The waist-hip ratio $(\mathrm{WHR})$ was calculated as: $\mathrm{WC}(\mathrm{cm}) / \mathrm{HC}$ $(\mathrm{cm})$.

\section{Questionnaires}

All subjects were asked to complete questionnaires exploring history of hypertension (yes or no) and/or diabetes (yes or no), and smoking and alcohol consumption status. Smoking status was divided into non-smoker, current smoker and ex-smoker; we also recorded the mean number of cigarettes smoked per day, smoking duration (years) and number of years since quitting (for ex-smokers). ${ }^{18}$ Non-smokers were defined as those who had either never smoked or had smoked for less than 12 months. Current smokers were those who self-reported cigarette use for at least 12 months. Ex-smokers were former smokers who had given up after smoking for at least 12 months and had remained abstinent for at least 12 months. We explored alcohol consumption status (non-drinkers, current drinkers and ex-drinkers). Ever-drinkers were considered to be alcohol consumers.

\section{Overnight PSG parameters}

Respiratory events were scored via laboratory-based PSG (Alice 4 or 5 platform; Respironics, Pittsburgh, Pennsylvania, USA). We obtained electroencephalographic, electro-oculographic, electrocardiographic and electromyographic data; nasal and oral airflows; thoracic and abdominal respiratory efforts; pulse oximetry data; information on posture; and snoring data. The apnoea-hypopnoea index (AHI) was the number of apnoea and hypopnoea events per hour during sleep. The oxygen desaturation index (ODI) was defined as the number of times per hour 
of sleep that the blood oxygen level dropped by $\geq 4 \%$ from baseline. The microarousal index (MAI) was the number of arousals per hour of sleep. AHIs of $\geq 5,>15$ and $>30$ events per hour were taken to reflect mild, moderate and severe OSA, respectively, in line with the criteria of the American Academy of Sleep Medicine. ${ }^{19}$

\section{Biochemical markers}

Fasting blood samples were drawn from the antecubital vein. Serum lipid profiles (including TC, HDL-C, LDL-C, fasting serum glucose and fasting serum insulin levels) were measured using routine procedures; serum glucose levels were measured with an H-7600 autoanalyser (Hitachi, Tokyo, Japan) and serum insulin levels via immunoradiology. The TC/HDL-C ratio was given by TC $(\mathrm{mmol} / \mathrm{L}) / \mathrm{HDL}-\mathrm{C}(\mathrm{mmol} / \mathrm{L})$. Insulin resistance was calculated using homeostasis model assessment (HOMA-IR) as follows: fasting serum insulin $(\mu \mathrm{U} / \mathrm{mL}) \times$ fasting plasma glucose $(\mathrm{mmol} / \mathrm{L}) / 22.5 .^{20}$ Hyper-TC, hypo-HDL-C and hyper-LDL-C status were defined as levels $>5.17 \mathrm{mmol} / \mathrm{L}$, $\leq 1.03 \mathrm{mmol} / \mathrm{L}$ and $\geq 3.37 \mathrm{mmol} / \mathrm{L}$, respectively; these are the diagnostic criteria of the Joint Committee for Developing Chinese Guidelines on Prevention and Treatment of Dyslipidemia in Adults. ${ }^{21}$ A hyper-TC/HDL-C ratio was defined as a ratio $\geq 4 .{ }^{2223}$

\section{Statistical methods}

Data are presented as means $\pm S D$, medians (with IQRs) or numbers (with percentages) if they were normally distributed, skewed or categorical, respectively. We dichotomised each sleep apnoea characteristic, with a high-level AHI defined as an AHI $>30$ and a high-level ODI and MAI defined as values above the medians. For each characteristic, we performed stratified analysis by dividing all patients into four groups by gender and the high (vs low) binary variable, and explored interactions between these two variables, as described previously. ${ }^{24}$ For example, when exploring interactions between gender and the ODI, we compared the ORs of females with ODIs $\leq 40.1$, females with ODIs $>40.1$, males with ODIs $\leq 40.1$ and males with ODIs $>40.1$. The group with the lowest risk served as the reference; we then calculated ORs for the other three groups and computed interactions. All models were adjusted for age, BMI, WHR, insulin level, smoking and alcohol consumption, diabetes, hypertension and MAI when analysing interactions between gender and AHI or ODI; and were adjusted for age, BMI, WHR, insulin level, smoking and alcohol consumption, diabetes, hypertension and ODI when analysing interactions between gender and MAI.

Additive biological interactions were measured using three indices: the relative excess risk due to interaction (RERI), the attributable proportion due to interaction (AP) and the synergy index (S). ${ }^{24}{ }^{25}$ A RERI or AP of 0 , and an S value of 1 , reflected no additive interaction. ${ }^{26}$ Multiplicative interaction was evaluated via logistic regression. Multiple linear regression was used to quantify the incremental value of each independent variable using the part-R-squares; we also calculated regression coefficients.
All statistical analyses were performed with SPSS (V.22.0) and R software (V.3.4.4; R Development Core Team, Vienna, Austria). A two-tailed $\mathrm{p}$ value $<0.05$ was considered to reflect statistical significance.

\section{RESULTS}

Ultimately, we analysed 3760 subjects: 638 females and 3122 males. The median ODI was 40.1 and the median MAI was 28.6 (table 1).

\section{Male and female risks of dyslipidaemia by AHI>30}

We divided the patients into four groups by gender and AHI: females with AHIs $\leq 30 \quad(n=377)$, females with AHIs $>30$ $(\mathrm{n}=261)$, males with $\mathrm{AHIs} \leq 30 \quad(\mathrm{n}=1128)$ and males with AHIs $>30 \quad(n=1994)$. After controlling for age, BMI, WHR, insulin level, smoking and alcohol consumption, diabetes, hypertension and MAI, we found that the risks of hyper-TC and hypo-HDL-C status, a hyper-TC/HDL-C ratio and hyper-LDL-C status for females with AHIs $>30$ were not significantly greater than those for females with AHIs $\leq 30$ $(\mathrm{OR}(95 \% \mathrm{CI})=1.358(0.948$ to 1.945$)$; OR $(95 \% \mathrm{CI})=1.006$ (0.683 to 1.483$)$; OR $(95 \% \mathrm{CI})=1.038$ (0.723 to 1.490$)$; and $\mathrm{OR}(95 \% \mathrm{CI})=1.233$ (0.850 to 1.787$)$, respectively) (tables 2-5).

In males, the risk of hyper-LDL-C status of those with AHIs $>30$ was not significantly higher than that for subjects with $\mathrm{AHIs} \leq 30 \quad(\mathrm{OR}(95 \% \mathrm{CI})=1.071 \quad(0.895$ to 1.282$))$, but the risks of hyper-TC and hypo-HDL-C status and a hyper-TC/HDL-C ratio were significantly higher for males with AHIs $>30$ (OR $(95 \% \mathrm{CI})=1.236$ (1.030 to 1.483); and $\mathrm{OR}(95 \% \mathrm{CI})=1.284(1.084$ to 1.520$)$; and OR $(95 \% \mathrm{CI})=1.332$ (1.100 to 1.612$)$, respectively) (tables $2-5$ ).

\section{Interaction between gender and AHI on dyslipidaemia}

After adjusting for age, BMI, WHR, insulin level, smoking and alcohol consumption, diabetes, hypertension and MAI, females with AHIs $>30$ (OR (95\% CI) $=2.239$ (1.648 to 3.039)) exhibited the highest OR for hyper-TC status of all groups; males with AHIs $\leq 30$ served as the reference group. The RERI (95\% CI) was 0.3297 ( -0.3488 to 1.008 ), indicating no statistically significant additive interaction between gender and AHI (table 2, figure 2). Males with AHIs $>30$ (OR (95\% CI)=3.012 (2.296 to 3.969)) exhibited the highest OR in terms of hypo-HDL-C status when females with AHIs $\leq 30$ served as the reference group. Although the $\mathrm{S}$ value (95\% CI) was 1.4012 (0.9626 to 2.0398$)$, the RERI (95\% CI) was 0.5761 (0.0520 to 1.1001$)$ and the AP (95\% CI) was 0.1913 (0.0178 to 0.3648$)$, indicating a positive additive interaction between gender and AHI (table 3 , figure 3). Males with AHIs $>30(\mathrm{OR}(95 \% \mathrm{CI})=2.031(1.544$ to 2.671) ) exhibited the highest OR for a hyper-TC/HDL-C ratio when females with $\mathrm{AHIs} \leq 30$ served as the reference group. Although the $\mathrm{S}$ value $(95 \% \mathrm{CI})$ was 1.9086 (0.7993 to $4.5569)$, the RERI (95\% CI) was 0.4910 (0.0610 to 0.9209$)$ and the AP (95\% CI) was 0.2417 (0.0295 to 0.4539$)$, indicating a positive additive interaction between male gender and AHI (table 4, figure 4). In terms of hyper-LDL-C 


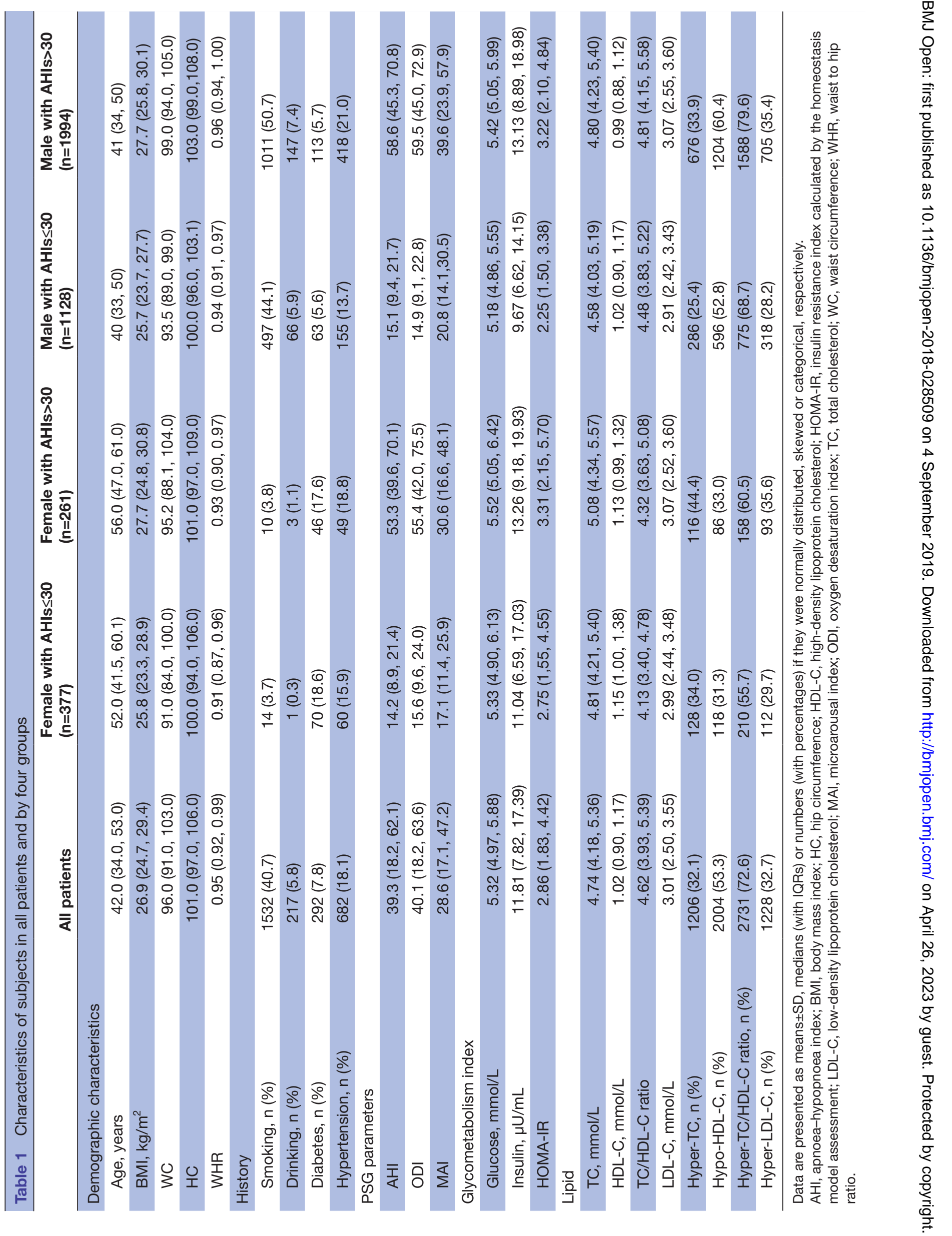


Table 2 Additive interaction between gender and $\mathrm{AHI}$ on the risk of hyper-TC

\begin{tabular}{|c|c|c|c|}
\hline & $\mathrm{AHI} \leq \mathbf{3 0}$ & $A H I>30$ & \multirow[b]{2}{*}{$\begin{array}{l}A H I>30 \text { vs } \\
A H I \leq 30\end{array}$} \\
\hline & $\begin{array}{l}\text { OR }(95 \% \mathrm{Cl}) \\
\mathrm{P} \text { value }\end{array}$ & $\begin{array}{l}\text { OR }(95 \% \mathrm{Cl}) \\
\mathrm{P} \text { value }\end{array}$ & \\
\hline Male & 1.0 & $\begin{array}{l}1.251(1.047 \text { to } \\
1.497) \\
p=0.014\end{array}$ & $\begin{array}{l}1.236(1.030 \text { to } \\
1.483) \\
p=0.023\end{array}$ \\
\hline Female & $\begin{array}{l}1.658(1.261 \text { to } \\
2.176) \\
p<0.001\end{array}$ & $\begin{array}{l}2.239 \text { (1.648 to } \\
3.039) \\
p<0.001\end{array}$ & $\begin{array}{l}1.358(0.948 \text { to } \\
1.945) \\
p=0.095\end{array}$ \\
\hline
\end{tabular}

Measure of effect modification on additive scale: RERI $(95 \% \mathrm{Cl})=0.3297(-0.3488$ to 1.008$) ; \mathrm{AP}(95 \% \mathrm{Cl})=0.1473(-0.1287$ to 0.4232$) ; \mathrm{S}(95 \% \mathrm{Cl})=1.3626(0.7224$ to 2.5703$)$. ORs were adjusted for age, BMI, WHR, insulin level, smoking and alcohol consumption, diabetes, hypertension and MAI. OR $(95 \% \mathrm{Cl})$ of diabetes in females $=0.960(0.594$ to 1.554$)$; OR $(95 \% \mathrm{Cl})$ of diabetes in males $=0.812$ ( 0.571 to 1.153 ).

$\mathrm{AHI}$, apnoea-hypopnoea index; AP, attributable proportion due to interaction; BMI, body mass index; MAI, microarousal index; RERI, relative excess risk due to interaction; $\mathrm{S}$, synergy index; TC, total cholesterol; WHR, waist to hip ratio.

status, the RERI (95\% CI) was 0.0149 ( -0.4305 to 0.4603$)$, indicating no statistically significant additive interaction between gender and AHI (table 5, figure 5).

After adjusting for confounding factors, no statistically significant multiplicative interaction between gender and AHI was found in terms of hyper-TC $($ OR $(95 \% \mathrm{CI})=1.002$ (0.995 to 1.010$))$, hyper-LDL-C (OR $(95 \% \mathrm{CI})=1.000(0.993$ to 1.007$)$ ), hyper-TC/HDL-C $(\mathrm{OR}(95 \% \mathrm{CI})=1.007(0.999$ to 1.015$)$ ) or hypo-HDL-C (OR $(95 \% \mathrm{CI})=1.001(0.994$ to 1.009)) status (table 6).

Table 3 Additive interaction between gender and $\mathrm{AHI}$ on the risk of hypo-HDL-C

\begin{tabular}{|c|c|c|c|}
\hline & \multirow{2}{*}{$\begin{array}{l}\mathrm{AHI} \leq 30 \\
\text { OR }(95 \% \mathrm{CI}) \\
\mathrm{P} \text { value }\end{array}$} & \multirow{2}{*}{$\begin{array}{l}\mathrm{AHI}>30 \\
\text { OR }(95 \% \mathrm{CI}) \\
\mathrm{P} \text { value }\end{array}$} & \multirow[b]{2}{*}{$\begin{array}{l}A H I>30 \text { vs } \\
A H I \leq 30\end{array}$} \\
\hline & & & \\
\hline Female & 1.0 & $\begin{array}{l}1.060(0.744 \text { to } \\
1.508) \\
p=0.745\end{array}$ & $\begin{array}{l}1.006(0.683 \text { to } \\
1.483) \\
p=0.975\end{array}$ \\
\hline Male & $\begin{array}{l}2.375 \text { (1.811 to } \\
3.131) \\
p<0.001\end{array}$ & $\begin{array}{l}3.012(2.296 \text { to } \\
3.969) \\
p<0.001\end{array}$ & $\begin{array}{l}1.284(1.084 \text { to } \\
1.520) \\
p=0.004\end{array}$ \\
\hline
\end{tabular}

Measure of effect modification on additive scale: RERI $(95 \% \mathrm{Cl})=0.5761(0.0520$ to 1.1001$) ; \mathrm{AP}(95 \% \mathrm{Cl})=0.1913(0.0178$ to 0.3648$)$; $\mathrm{S}(95 \% \mathrm{Cl})=1.4012$ (0.9626 to 2.0398$)$; ORs were adjusted for age, BMI, WHR, insulin level, smoking and alcohol consumption, diabetes, hypertension and MAI. OR $(95 \% \mathrm{Cl})$ of diabetes in females $=0.782(0.474$ to 1.292$)$; OR $(95 \% \mathrm{Cl})$ of diabetes in males $=1.677$ (1.183 to 2.377$)$.

$\mathrm{AHI}$, apnoea-hypopnoea index; AP, attributable proportion due to interaction; BMI, body mass index; HDL-C, high-density lipoprotein cholesterol; MAI, microarousal index; RERI, relative excess risk due to interaction; S, synergy index; WHR, waist to hip ratio.
Table 4 Additive interaction between gender and $\mathrm{AHI}$ on the risk of hyper-TC/HDL-C ratio

\begin{tabular}{|c|c|c|c|}
\hline & $\mathrm{AHI} \leq 30$ & $A H I>30$ & \multirow[b]{2}{*}{$\begin{array}{l}A H I>30 \text { vs } \\
A H I \leq 30\end{array}$} \\
\hline & $\begin{array}{l}\text { OR }(95 \% \mathrm{Cl}) \\
\mathrm{P} \text { value }\end{array}$ & $\begin{array}{l}\text { OR }(95 \% \mathrm{Cl}) \\
\mathrm{P} \text { value }\end{array}$ & \\
\hline Female & 1.0 & $\begin{array}{l}1.034(0.737 \text { to } \\
1.452) \\
p=0.848\end{array}$ & $\begin{array}{l}1.038(0.723 \text { to } \\
1.490) \\
p=0.838\end{array}$ \\
\hline Male & $\begin{array}{l}1.507(1.151 \text { to } \\
1.972) \\
p=0.003\end{array}$ & $\begin{array}{l}2.031(1.544 \text { to } \\
2.671) \\
p<0.001\end{array}$ & $\begin{array}{l}1.332(1.100 \text { to } \\
1.612) \\
p=0.003\end{array}$ \\
\hline
\end{tabular}

Measure of effect modification on additive scale: RERI $(95 \% \mathrm{Cl})=0.4910(0.0610$ to 0.9209$) ; \mathrm{AP}(95 \% \mathrm{Cl})=0.2417(0.0295$ to 0.4539$)$; $\mathrm{S}(95 \% \mathrm{Cl})=1.9086$ (0.7993 to 4.5569$)$. ORs were adjusted for age, BMI, WHR, insulin level, smoking and alcohol consumption, diabetes, hypertension and MAI. OR $(95 \% \mathrm{Cl})$ of diabetes in females $=1.099(0.667$ to 1.812$)$; OR $(95 \% \mathrm{Cl})$ of diabetes in males $=0.924$ (0.628 to 1.359).

$\mathrm{AHI}$, apnoea-hypopnoea index; AP, attributable proportion due to interaction; $\mathrm{BMI}$, body mass index; HDL-C, high-density lipoprotein cholesterol; MAI, microarousal index; RERI, relative excess risk due to interaction; S, synergy index; TC, total cholesterol; WHR, waist to hip ratio.

Male and female risks of dyslipidaemia by the median ODI All subjects were divided into four groups: females with ODIs $\leq 40.1 \quad(n=422)$, females with ODIs $>40.1 \quad(n=216)$, males with ODIs $\leq 40.1 \quad(n=1459)$ and males with ODIs $>40.1$ $(\mathrm{n}=1663)$. After controlling for age, BMI, WHR, insulin level, smoking and alcohol consumption, diabetes, hypertension and MAI, the risks of hyper-TC, hypo-HDL-C, hyper-TC/HDL-C ratio and hyper-LDL-C status for females with ODIs $>40.1$ were not significantly higher than those for females with ODIs $\leq 40.1$. In males, the risks of hyper-TC and hyper-LDL-C status of subjects with ODIs $>40.1$ were not

Table 5 Additive interaction between gender and $\mathrm{AHI}$ on the risk of hyper-LDL-C

\begin{tabular}{|c|c|c|c|}
\hline & \multirow{2}{*}{$\begin{array}{l}A H I \leq 30 \\
\text { OR }(95 \% \mathrm{CI}) \\
\text { P value }\end{array}$} & \multirow{2}{*}{$\begin{array}{l}\text { AHI>30 } \\
\text { OR }(95 \% \mathrm{CI}) \\
\text { P value }\end{array}$} & \multirow[b]{2}{*}{$\begin{array}{l}A H I>30 \text { vs } \\
A H I \leq 30\end{array}$} \\
\hline & & & \\
\hline Male & 1.0 & $\begin{array}{l}1.096(0.919 \text { to } \\
1.307) \\
p=0.307\end{array}$ & $\begin{array}{l}1.071(0.895 \\
\text { to1.282) } \\
\mathrm{p}=0.455\end{array}$ \\
\hline Female & $\begin{array}{l}1.150(0.870 \text { to } \\
1.513) \\
p=0.323\end{array}$ & $\begin{array}{l}1.260(0.920 \text { to } \\
1.720) \\
p=0.147\end{array}$ & $\begin{array}{l}1.233(0.850 \\
\text { to1.787) } \\
\mathrm{p}=0.270\end{array}$ \\
\hline
\end{tabular}

Measure of effect modification on additive scale: RERI $(95 \% \mathrm{Cl})=0.0149(-0.4305$ to 0.4603$) ; \mathrm{AP}(95 \% \mathrm{Cl})=0.0118$ $(-0.3397$ to 0.3634$)$; S $(95 \% \mathrm{Cl})=1.0607$ (0.1806 to 6.2285$)$. ORs were adjusted for age, BMI, WHR, insulin level, smoking and alcohol consumption, diabetes, hypertension and MAI. OR $(95 \% \mathrm{Cl})$ of diabetes in females $=1.044$ (0.638 to 1.710$)$. OR $(95 \% \mathrm{Cl})$ of diabetes in males $=0.535$ (0.367 to 0.781$)$.

$\mathrm{AHI}$, apnoea-hypopnoea index; AP, attributable proportion due to interaction; BMI, body mass index; LDL-C, low-density lipoprotein cholesterol; MAI, microarousal index; RERI, relative excess risk due to interaction; S, synergy index; WHR, waist to hip ratio. 
Additive interaction between gender and $\mathrm{AHI}$ on the risk of hyper-TC

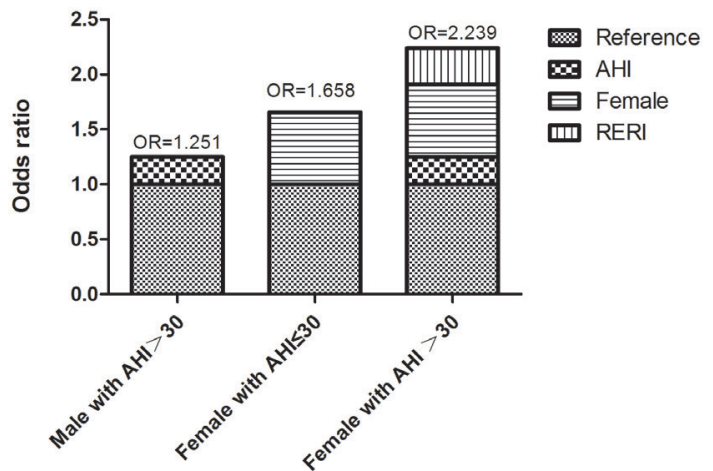

Figure 2 Additive interaction between gender and $\mathrm{AHI}$ on the risk of hyper-TC. AHI, apnoea-hypopnoea index; TC, total cholesterol; Ref, reference; RERI, relative excess risk due to interaction.

significantly higher than those of males with ODIs $\leq 40.1$, but the risks of hypo-HDL-C status and a hyper-TC/HDL-C ratio were significantly higher in the group with ODIs $>40.1$ (online supplementary tables 1-4).

\section{Interactions between gender and ODI on dyslipidaemia}

After adjusting for confounding factors, no statistically significant additive or multiplicative interaction between gender and ODI was apparent in terms of any of hyper-TC, hypo-HDL-C or hyper-LDL-C status (online supplementary tables $1-5)$. Although the $\mathrm{S}$ value $(95 \% \mathrm{CI})$ was 2.2656 (0.9214 to 5.5710), the RERI (95\% CI) was 0.6686 ( 0.2031 to $1.1342)$ and the AP (95\% CI) was 0.3043 (0.1045 to 0.5042$)$, indicating positive additive interactions between male gender and ODI (online supplementary table 3). A positive multiplicative interaction of male gender and ODI on a hyper-TC/HDL-C ratio was evident $(\mathrm{OR}(95 \% \mathrm{CI})=1.008$ (1.001 to 1.016)) (online supplementary table 5).

\section{Male and female risks of dyslipidaemia by median MAI}

We divided the patients into four groups by gender and median MAI: females with MAIs $\leq 28.6(n=426)$, females with MAIs $>28.6(n=212)$, males with MAIs $\leq 28.6 \quad(n=1456)$ and males with MAIs $>28.6 \quad(n=1666)$. In females, no

Additive interaction between gender and AHI on the risk of hypo-HDL-C

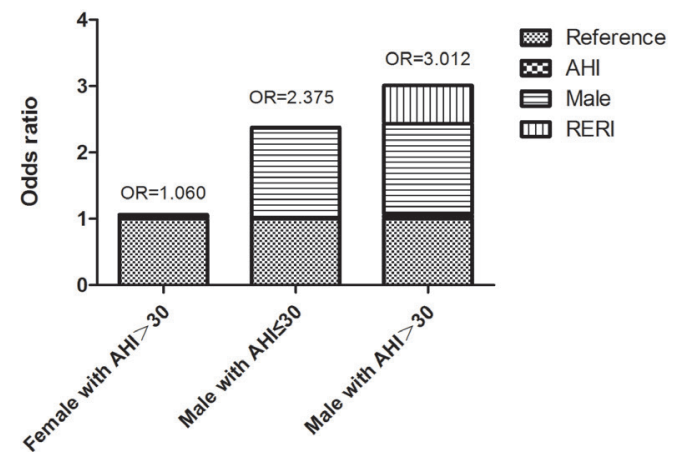

Figure 3 Additive interaction between gender and $\mathrm{AHI}$ on the risk of hypo-HDL-C. AHI, apnoea-hypopnoea index; HDL-C, high-density lipoprotein cholesterol; Ref, reference; $\mathrm{RERI}$, relative excess risk due to interaction.
Additive interaction between gender and $\mathrm{AHI}$ on the risk of hyper-TC/HDL-C ratio

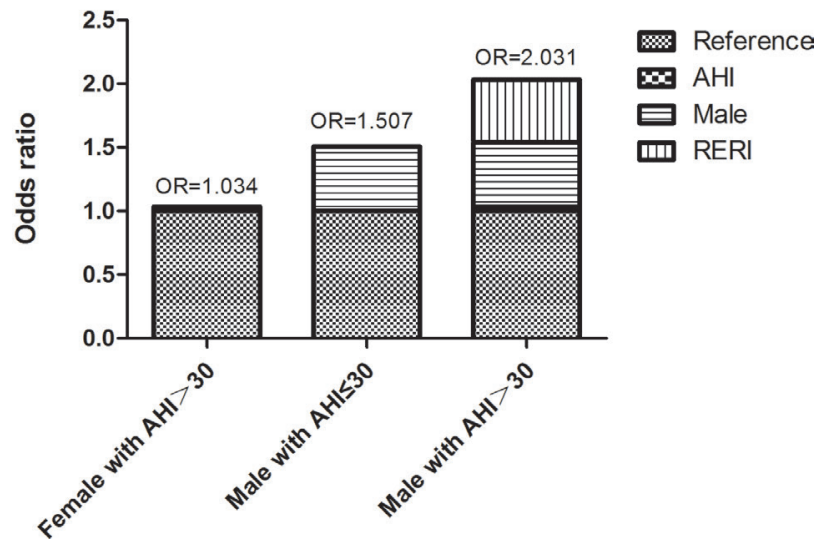

Figure 4 Additive interaction between gender and $\mathrm{AHI}$ on the risk of hyper-TC/HDL-C ratio. AHI, apnoea-hypopnoea index;TC, total cholesterol; HDL-C, high-density lipoprotein cholesterol; Ref, reference; RERI, relative excess risk due to interaction.

significant difference in the risk of hyper-TC, hypo-HDL-C, hyper-TC/HDL-C ratio or hyper-LDL-C status was evident between those with MAIs $>28.6$ and $\leq 28.6$. In males, those with MAIs $>28.6$ were at significantly greater risk of hyper-TC and hyper-LDL-C status than those with MAIs $\leq 28.6$, but no significant difference in the risk of either hypo-HDL-C or hyper TC/HDL-C ratio status was evident (online supplementary tables 6-9).

\section{Interaction between gender and MAI on dyslipidaemia}

After adjusting for age, BMI, WHR, insulin level, smoking and alcohol consumption, diabetes, hypertension and ODI, no statistically significant additive interaction between gender and MAI on hyper-TC, hypo-HDL-C, hyper-TC/ HDL-C ratio or hyper-LDL-C status was apparent (online supplementary tables 6-9). After adjusting for confounding factors, no statistically significant multiplicative interaction between gender and MAI in terms of hyper-TC or hypoHDL-C status, hyper-TC/HDL-C ratio or hyper-LDL-C

Additive interaction between gender and AHI on the risk of hyper-LDL-C

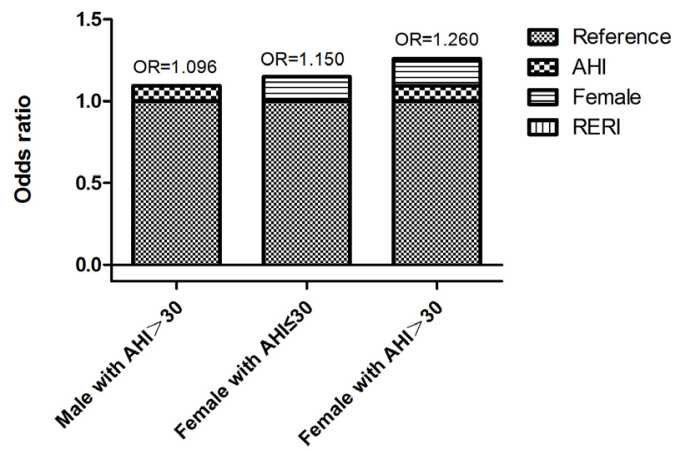

Figure 5 Additive interaction between gender and $\mathrm{AHI}$ on the risk of hyper-LDL-C. AHI, apnoea-hypopnoea index; LDL-C, low-density lipoprotein cholesterol; Ref, reference; RERI, relative excess risk due to interaction. 
status was evident (all $\mathrm{p}$ values for gender*MAIs $>0.05$ ) (online supplementary table 10).

\section{Subgroup analysis of subjects aged $\geq 55$ years}

In total, 829 patients with OSA were aged $\geq 55$ years. For both females and males, no significant difference in the risk of either hyper-TC or hypo-HDL-C status, the hyper-TC/ HDL-C ratio or hyper-LDL-C status was evident between groups divided by AHI, ODI or MAI described above (online supplementary tables 11-22).

In those aged $\geq 55$ years, positive additive and multiplicative interactions between the male gender and the AHI on a hyper-TC/HDL-C ratio were evident (RERI $(95 \% \mathrm{CI})=0.6828 \quad(0.0925$ to 1.2730$)$; OR $(95 \% \mathrm{CI})$ for male*AHI $=1.017 \quad(1.003,1.031)$, respectively) (online supplementary tables 13 and 23). Positive additive and multiplicative interactions between the male gender and the ODI on a hyper-TC/HDL-C ratio were apparent (RERI $(95 \% \mathrm{CI})=0.6690 \quad(0.1015$ to 1.2364$)$; OR $(95 \% \mathrm{CI})$ for male*ODI=1.018 (1.005 to 1.032) ) (online supplementary tables 17 and 24). No statistically significant interaction between gender and MAI on dyslipidaemia was evident (online supplementary tables 19-22 and 25).

\section{Subgroup analysis of the regression coefficients of HDL-C levels}

To estimate regression coefficients for each factor associated with HDL-C, age, BMI, WHR, insulin level, smoking and alcohol consumption, diabetes, hypertension, ODI and MAI were subjected to multiple linear regression. In males, the adjusted associations of HDL-C levels included BMI $\left(\beta=-0.137, R^{2}=0.030\right)$, drinking $\left(\beta=0.107, R^{2}=0.012\right)$, smoking $\left(\beta=-0.103, R^{2}=0.009\right)$, hypertension $(\beta=0.076$, $\left.R^{2}=0.007\right)$, insulin $\left(\beta=-0.067, R^{2}=0.005\right)$, age $(\beta=0.057$, $\left.\mathrm{R}^{2}=0.002\right)$ and diabetes $\left(\beta=-0.049, \mathrm{R}^{2}=0.002\right)$. In females, the adjusted associations of HDL-C levels were insulin $\left(\beta=-0.178, R^{2}=0.078\right)$, hypertension $\left(\beta=0.131, R^{2}=0.020\right)$, BMI $\left(\beta=-0.090, R^{2}=0.016\right)$, WHR $\left(\beta=-0.124, R^{2}=0.006\right)$ and age $\left(\beta=0.112, R^{2}=0.011\right)$ (online supplementary table 26 ).

\section{DISCUSSION}

After controlling for confounding factors, we found that males (but not females) with severe OSA (AHI $>30$ ) were at higher risks of hyper-TC and hypo-HDL-C status and a hyper-TC/HDL-C ratio than those with $A H I s \leq 30$. Positive additive interactions were evident between male gender and AHI on hypo-HDL-C status and a hyper-TC/HDL-C ratio. Further analysis revealed that males with ODIs $>40.1$ were at higher risk of hypo-HDL-C and hyper-TC/HDL-C ratio status than those with ODIs $\leq 40.1$. Positive additive and multiplicative interactions were evident between male gender and ODI on hyper-TC/HDL-C ratio status (online supplementary tables 3 and 5). Only males with MAIs $>28.6$ were at higher risk of hyper-TC and hyper-LDL-C status than those with MAIs $\leq 28.6$, but no statistically significant additive or multiplicative interaction between gender and MAI in terms of dyslipidaemia was apparent. Subgroup analysis revealed that for those aged $\geq 55$ years, although the significant differences 
in terms of the risk of dyslipidaemia between groups divided by AHI, ODI or MAI disappeared in males, the interactions between male gender and AHI or ODI on some measures of dyslipidaemia remained. In terms of the HDL-C level, independent factors affecting this level included obesity, hypertension, alcohol consumption, smoking, diabetes, age and insulin level in males; and insulin level, hypertension, obesity and age in females.

We found that severe OSA (as reflected by an AHI $>30$ ) was associated with increased risks of hyper-TC, hypo-HDL-C status and a hyper-TC/HDL-C ratio, but only in males (not in females). One of the most significant pathophysiological characteristics of OSA, intermittent hypoxia (as reflected by the ODI), was also associated with increased hypoHDL-C status and a hyper-TC/HDL-C ratio in males but not females. We found positive additive interactions (synergistic effects) between male gender and AHI on hypo-HDL-C status and a hyper-TC/HDL-C ratio, and positive multiplicative and additive interactions between male gender and ODI on a hyper-TC/HDL-C ratio, suggesting that maleness augments the effect of severe OSA or intermittent hypoxia on certain measures of dyslipidaemia. Thus, the impact of severe OSA or intermittent hypoxia on dyslipidaemia was more obvious in males than females. Several possible explanations for this may be advanced. First, OSA develops early and is more severe in males, who exhibit more typical symptoms, more obvious oxygen desaturation and longer apnoea durations. ${ }^{11-13}$ Males have higher rates of OSA than females of the same AHI. ${ }^{15}{ }^{16}$ Second, oestrogen may protect females from dyslipidaemia. Intermittent hypoxia increases the liver level of hypoxia-inducible factor-1 (HIF-1 $\alpha$ ), in turn, activating the sterol regulatory element binding protein 1 and stearoyl-coenzyme desaturase-1, which act to increase liver fatty acid synthesis, in turn, elevating the levels of lipids such as TG and cholesterol. ${ }^{27}{ }^{28}$ Earlier rodent studies revealed that oestrogen downregulated HIF-1 $\alpha$ production, ${ }^{29}$ thus curtailing the effects of intermittent hypoxia on dyslipidaemia in females. Third, males with OSA have more visceral adipose tissue (VAT) than females with OSA, although BMIs and WCs are similar ${ }^{14}$; excess VAT is closely associated with an atherogenic dyslipidaemic profile. ${ }^{30}{ }^{31}$ Our earlier clinical study revealed that in females with OSA, age, obesity/central obesity and glucose metabolism were major risk factors for elevations in the serum levels of most lipids, and were major determinants of dyslipidaemia after multiple adjustments; OSA had only a limited effect on dyslipidaemia in females. ${ }^{32}$ Thus, severe OSA and intermittent hypoxia affects dyslipidaemia principally in males.

Although we did not find any statistically significant additive or multiplicative interactions between gender and MAI (sleep fragmentation) in terms of dyslipidaemia, males with MAIs $>28.6$ were at higher risk of hyper-TC and hyperLDL-C status than those with MAIs $\leq 28$.6, indicating that sleep fragmentation affected dyslipidaemia. A previous rodent study found an association between sleep fragmentation in late-stage pregnant mice and dyslipidaemia only in male offspring. ${ }^{33}$ As is true of intermittent hypoxia, sleep fragmentation affected males more than females. Sleep fragmentation is associated with autonomic (sympathetic) nervous system activation, the sleep-related autonomic activation index is correlated with an increased LDL-C level, which may explain the link between sleep fragmentation and hyper-LDL-C status, ${ }^{34}$ but the underlying mechanism of effect modification by gender of sleep fragmentation in terms of dyslipidaemia remains unclear.

In subjects aged $\geq 55$ years, the statistically significant differences (in younger subjects) in the risks of dyslipidaemia between groups divided by AHI, ODI or MAI disappeared in males, which may (in part) reflect the relatively small number of participants in this category. The interactions between male gender and AHI or ODI in terms of some measures of dyslipidaemia remained; severe OSA or intermittent hypoxia increased some measures of dyslipidaemia in males. Thus, OSA in older males should not be ignored when treating dyslipidaemia. We found that diabetes was positively associated with hypo-HDL-C status (OR $(95 \% \mathrm{CI})=1.677$ (1.183 to 2.377$)$ ) (table 3 ) and negatively linked to hyper-LDL-C status (OR (95\% CI) $=0.535$ (0.367 to 0.781 ) ) (table 5) in males, but was not independently associated with dyslipidaemia in females (tables 2-5). We also analysed effects on HDL-C levels. Although such levels are influenced by many factors, the major determinant was BMI (with the largest $\mathrm{R}^{2}$ value: 0.030 ) in males, and insulin level (with the largest $\mathrm{R}^{2}$ value: 0.078 ) in females (online supplementary table 26). Thus, obesity and diabetes in males, and insulin resistance in females, should be addressed when seeking to control HDL-C levels. However, the negative association between diabetes and hyper-LDL-C status that we found contradicts the result of previous studies ${ }^{35}$; further work is required.

Our study had certain limitations. First, although we adjusted for several common confounding factors, other more complex factors, such as lifestyle choices, exercise status and dietary habits, were not considered. Interaction analysis cannot reveal any essential difference between the genders. Second, our study used an observational hospital-based design, rather than a prospective or community-based design. Third, the sleep architecture abnormalities noted may have been caused, at least in part, by the 'first night effect' during the single night of PSG monitoring. ${ }^{36}$ Finally, we did not classify the lipid-lowering drugs used; various drugs were prescribed. Despite these limitations, the sleep data and relatively large sample size enhance the credibility of the results.

In conclusion, in males with severe OSA, a higher ODI or MAI was associated with a greater risk of certain forms of dyslipidaemia. Positive additive interactions were evident between male gender and AHI on hypo-HDL-C status and a hyper-TC/HDL-C ratio. Positive additive and multiplicative interactions were also apparent between male gender and intermittent hypoxia on a hyper-TC/ HDL-C ratio. However, we found no statistically significant additive or multiplicative interaction between gender and MAI in terms of dyslipidaemia. Thus, dyslipidaemia status should be evaluated in patients with OSA, especially males with severe OSA or intermittent hypoxia. 
Author affiliations

${ }^{1}$ Department of Otolaryngology Head and Neck Surgery \& Center of Sleep Medicine, Shanghai Jiao Tong University Affiliated Sixth People's Hospital, Shanghai, China

${ }^{2}$ Department of otorhinolaryngology Head and Neck Surgery, First Affiliated Hospital of Nanchang University, Nangchang, China

${ }^{3}$ Shanghai Key Laboratory of Sleep Disordered Breathing, Shanghai Jiao Tong University Affiliated Sixth People's Hospital, Shanghai, China

${ }^{4}$ Otolaryngological Institute of Shanghai Jiao Tong University, Shanghai Jiao Tong University Affiliated Sixth People's Hospital, Shanghai, China

Contributors YX, JZ, HX and JG provided the conceptual design of the project, writing and editing the final version of the manuscript. HY and SY participated in writing and editing the final version of the manuscript. All listed authors read and approved the final manuscript. Also, we thanked the patient advisers for contributing to the study.

Funding This work was supported by the Science and Technology Commission of Shanghai Municipality, China (Grant Nos. 16411950104, 16411950105 and 16411950106).

\section{Competing interests None declared.}

\section{Patient consent for publication Obtained.}

Ethics approval The study protocol was approved by the Internal Review Board of the Institutional Ethics Committee of Shanghai Jiao Tong University Affiliated Sixth People's Hospital and the study was conducted in accordance with all relevant tenets of the Declaration of Helsinki.

\section{Provenance and peer review Not commissioned; externally peer reviewed.}

Data availability statement All authors had access to and take responsibility for the data and analyses. Relevant data can be available for research on reasonable request (sunnydayxu2010@163.com). Data will be saved for 5 years.

Open access This is an open access article distributed in accordance with the Creative Commons Attribution Non Commercial (CC BY-NC 4.0) license, which permits others to distribute, remix, adapt, build upon this work non-commercially, and license their derivative works on different terms, provided the original work is properly cited, appropriate credit is given, any changes made indicated, and the use is non-commercial. See: http://creativecommons.org/licenses/by-nc/4.0/.

\section{REFERENCES}

1. Heinzer R, Vat S, Marques-Vidal P, et al. Prevalence of sleep-disordered breathing in the general population: the HypnoLaus study. Lancet Respir Med 2015;3:310-8.

2. Newman AB, Nieto FJ, Guidry U, et al. Relation of sleep-disordered breathing to cardiovascular disease risk factors: the sleep heart health study. Am J Epidemiol 2001;154:50-9.

3. Roche F, Sforza E, Pichot V, et al. Obstructive sleep apnoea/hypopnea influences high-density lipoprotein cholesterol in the elderly. Sleep Med 2009;10:882-6.

4. Barthélémy J-C, Pichot V, Dauphinot V, et al. Autonomic nervous system activity and decline as prognostic indicators of cardiovascular and cerebrovascular events: the 'PROOF' study. Study design and population sample. Associations with sleep-related breathing disorders: the 'SYNAPSE 'Study. Neuroepidemiology 2007;29:18-28.

5. Börgel J, Sanner BM, Bittlinsky A, et al. Obstructive sleep apnoea and its therapy influence high-density lipoprotein cholesterol serum levels. Eur Respir J 2006;27:121-7.

6. Togeiro SM, Carneiro G, Ribeiro Filho FF, et al. Consequences of obstructive sleep apnea on metabolic profile: a population-based survey. Obesity 2013;21:847-51.

7. Toyama Y, Chin K, Chihara Y, et al. Association between sleep apnea, sleep duration, and serum lipid profile in an urban, male, working population in Japan. Chest 2013;143:720-8.

8. Qian Y, Yi H, Zou J, et al. Independent association between sleep fragmentation and dyslipidemia in patients with obstructive sleep apnea. Sci Rep 2016;6:26089.

9. Trzepizur W, Le Vaillant M, Meslier N, et al. Independent association between nocturnal intermittent hypoxemia and metabolic dyslipidemia. Chest 2013;143:1584-9.

10. Guan J, Yi H, Zou J, et al. Distinct severity stages of obstructive sleep apnoea are correlated with unique dyslipidaemia: large-scale observational study. Thorax 2016;71:347-55.

11. Won C, Guilleminault C. Gender differences in sleep disordered breathing: implications for therapy. Expert Rev Respir Med 2015;9:221-31.
12. Nigro CA, Dibur E, Borsini E, et al. The influence of gender on symptoms associated with obstructive sleep apnea. Sleep Breath 2018;22:683-93.

13. Subramanian $S$, Hesselbacher S, Mattewal A, et al. Gender and age influence the effects of slow-wave sleep on respiration in patients with obstructive sleep apnea. Sleep Breath 2013;17:51-6.

14. Harada Y, Oga T, Chihara Y, et al. Differences in associations between visceral fat accumulation and obstructive sleep apnea by sex. Ann Am Thorac Soc 2014;11:383-91.

15. Roca GQ, Redline S, Claggett B, et al. Sex-specific association of sleep apnea severity with subclinical myocardial injury, ventricular hypertrophy, and heart failure risk in a community-dwelling cohort: the atherosclerosis risk in communities-sleep heart health study. Circulation 2015;132:1329-37.

16. Minai OA, Ricaurte $B$, Kaw R, et al. Frequency and impact of pulmonary hypertension in patients with obstructive sleep apnea syndrome. Am J Cardiol 2009;104:1300-6.

17. Toyama Y, Tanizawa K, Kubo T, et al. Impact of obstructive sleep apnea on liver fat accumulation according to sex and visceral obesity. PLOS One 2015;10:e0129513.

18. Lin YN, Zhou LN, Zhang XJ, et al. Combined effect of obstructive sleep apnea and chronic smoking on cognitive impairment. Sleep Breath 2016;20:51-9.

19. Berry RB, Budhiraja R, Gottlieb DJ, et al. Rules for scoring respiratory events in sleep: update of the 2007 AASM manual for the scoring of sleep and associated events. deliberations of the sleep apnea definitions Task force of the American Academy of sleep medicine. $J$ Clin Sleep Med 2012;8:597-619.

20. Matthews DR, Hosker JP, Rudenski AS, et al. Homeostasis model assessment: insulin resistance and beta-cell function from fasting plasma glucose and insulin concentrations in man. Diabetologia 1985;28:412-9.

21. Jcf A, Dcgo P, To D, et al. [Chinese guidelines on prevention and treatment of dyslipidemia in adults]. Zhonghua Xin Xue Guan Bing Za Zhi 2007;35:390-419.

22. McPherson R, Frohlich J, Fodor G, et al. Canadian cardiovascular Society position statement - recommendations for the diagnosis and treatment of dyslipidemia and prevention of cardiovascular disease. Can J Cardiol 2006;22:913-27.

23. Millán J, Pintó X, Muñoz A, et al. Lipoprotein ratios: physiological significance and clinical usefulness in cardiovascular prevention. Vasc Health Risk Manag 2009;5:757-65.

24. Knol MJ, VanderWeele TJ. Recommendations for presenting analyses of effect modification and interaction. Int J Epidemiol 2012;41:514-20.

25. Andersson T, Alfredsson L, Källberg H, et al. Calculating measures of biological interaction. Eur J Epidemiol 2005;20:575-9.

26. Knol MJ, VanderWeele TJ, Groenwold RHH, et al. Estimating measures of interaction on an additive scale for preventive exposures. Eur $J$ Epidemiol 2011;26:433-8.

27. Li J, Grigoryev DN, Ye SQ, et al. Chronic intermittent hypoxia upregulates genes of lipid biosynthesis in obese mice. $J$ Appl Physiol 2005;99:1643-8.

28. Li J, Savransky V, Nanayakkara A, et al. Hyperlipidemia and lipid peroxidation are dependent on the severity of chronic intermittent hypoxia. J Appl Physiol 2007;102:557-63.

29. Xu J, Xiang Q, Lin G, et al. Estrogen improved metabolic syndrome through down-regulation of VEGF and HIF-1 $\alpha$ to inhibit hypoxia of periaortic and intra-abdominal fat in ovariectomized female rats. $\mathrm{Mol}$ Biol Rep 2012;39:8177-85.

30. Fox CS, Massaro JM, Hoffmann U, et al. Abdominal visceral and subcutaneous adipose tissue compartments: association with metabolic risk factors in the Framingham heart study. Circulation 2007;116:39-48.

31. Oka R, Miura K, Sakurai M, et al. Impacts of visceral adipose tissue and subcutaneous adipose tissue on metabolic risk factors in middle-aged Japanese. Obesity 2010;18:153-60.

32. Xia Y, Fu Y, Wang Y, et al. Prevalence and predictors of atherogenic serum lipoprotein dyslipidemia in women with obstructive sleep apnea. Sci Rep 2017;7:41687.

33. Khalyfa A, Carreras A, Almendros I, et al. Sex dimorphism in late gestational sleep fragmentation and metabolic dysfunction in offspring mice. Sleep 2015;38:545-57.

34. Crawford-Achour E, Roche F, Pichot V, et al. Sleep-related autonomic overactivity in a general elderly population and its relationship to cardiovascular regulation. Heart Vessels 2016;31:46-51.

35. Feingold KR, Grunfeld C. Diabetes and Dyslipidemia. In: Endotext. South Dartmouth (MA): MDText.com, Inc, 2019.

36. Santos-Silva R, Sartori DE, Truksinas V, et al. Validation of a portable monitoring system for the diagnosis of obstructive sleep apnea syndrome. Sleep 2009;32:629-36. 